\title{
El chamanismo y las drogas enteogénicas/alucinatorias del mundo precolombino
}

\section{Shamanism and Entheogenic/Hallucinatory Drugs in the Pre-Columbian World}

\author{
Carlos M. Quirce Balma* \\ Universidad de Costa Rica \\ (recibido 11 de noviembre de 2010; aceptado 15 Marzo de 2011)
}

\begin{abstract}
Resumen
Este trabajo intenta abordar algunas de las teorías más modernas y prevalentes sobre el chamanismo. Se han incluido tanto las teorías tradicionales como las modernas que parecen indicar las tendencias interpretativas de tipo neurofisiológico sobre los estados alterados y superiores de la conciencia. Se ha mencionado el uso prevalente y central de los diversos psicodélicos y plantas que contienen sustancias alterantes entre las diversas culturas precolombinas. Los trabajos de la antropología psicodélica han sido incluidos y su contraste con las escuelas influenciadas por Mircea Eliade.
\end{abstract}

Palabras clave: chamanismo, plantas psicodélicas, brujería, neurofisiología de la conciencia

\begin{abstract}
This article presents some of the most current and widespread theories on shamanism. Both traditional and modern theories which lean towards neurophysiologic interpretations of altered and higher states of consciousness are dealt with. The generalized and central use of psychedelics and plants containing altering substances by the different preColumbian cultures are discussed. Moreover, work by psychedelic anthropologists has been contrasted with the schools of thought influenced by Mircea Eliade.
\end{abstract}

Keywords: shamanism, psychedelic plants, witchcraft, neurophysiology of consciousness

\footnotetext{
* Escuela de Psicología, Facultad de Ciencias Sociales, Facultad de Farmacia, Universidad de Costa Rica. Correspondencia. Correo Electrónico: carlosmqb@gmail.com

ISSN 0257-1439

(C) Colegio Profesional de Psicólogos de Costa Rica http://www.revistacostarricensedepsicologia.com/
} 


\section{El chamanismo}

El chamanismo es quizás la religión más antigua del mundo. Está profundamente unida -si se quiere identificada- con la herbología alucinatoria, o aún mejor, la herbología enteogénica. Sus características mundiales y particularmente precolombinas son las de: (a) adivinación y magia de climas y agricultura, (b) contacto con el mundo de los ancestros, (c) magia de la cacería, (d) herbología y medicina indígena o nativa, (e) rituales, ritos, oraciones y sacrificios para atraer la lluvia y el buen tiempo y echar el mal tiempo de la agricultura, (f) el uso de diseños que contienen los cuatro puntos cardenales y un centro -entre los Navajo de los Estados Unidos y los aztecas cada punto direccional era representado con un color distinto. El diseño de la swasta que, según Campbell (1959), nace en el lago Baikal en Rusia, puede ser el responsable de esa costumbre.

Otros creen que los indígenas precolombinos conocían los cuatro puntos cardenales en la forma magnética mucho antes de la llegada de los españoles y que conocían el compás. Algunos autores han sugerido que lo utilizaban para navegar y para viajar largas distancias. Otras características del chamanismo son ( $\mathrm{g}$ ) el uso de diseños basados en las religiones derivadas del dios rey Quetzalcóatl que comprenden tanto la culebra como las alas de un ave, (h) el contacto con el mundo de los dioses a través de una herbología enteogénica, danzas y música ritualística adecuada para ello, (i) sacrificios de partes de las cosechas con la finalidad de traer bendiciones del cielo y del mundo de los ancestros, (j) servir a maestros de parte de nuevos aspirantes a chamanes, (k) dirigir obras de arte, música y teatro con la finalidad de impartir lecciones y obtener visiones y $(\mathrm{m})$ defender a las personas de los ataques de brujos, otros chamanes y espíritus malignos.

Mucho del chamanismo utiliza las drogas o plantas enteogénicas o alternativamente plantas de acción anticolinérgicas, para entrar en el mundo de los dioses y ancestros. En ese sentido, Schultes y Hofman (1979) ha llamado dichas plantas, las plantas de los dioses. El chamanismo se caracteriza por ser un sacerdocio de muchas religiones alucinatorias. No avoca el uso recreacional de ellas mismas. Los diversos tipos de alucinógenos son los sacramentos de dicha religión. De nuevo, una de las funciones del chamanismo es entrenar en esa sabiduría al nuevo discípulo que algún ha de llegar a ser el chamán de la tribu.

\section{La influencia de Wasson sobre el chamanismo}

Harner (1977a, 1977b, 1977c) distingue entre el chamán que usa sus conocimientos para herir o matar y el que solamente lo usa para curar y ayudar. Después de un largo estudio en México y otros países, tales como Ecuador, Venezuela y Colombia en el área amazónica, Harner concluye que los chamanes que curan y que ayudan son los de más alto nivel y los otros son generalmente los que no terminaron sus estudios con su maestro. Algunas de las principales etapas de desarrollo, en la teoría antropológica, sobre el chamanismo, son la consecuencia de una serie de cambios fundamentales, que en realidad datan desde la obra de Eliade (1966), hasta llegar en tiempos modernos a influenciar a autores como Ott (1993).

En el periodo en que Eliade dominó la interpretación en el campo de la antropología nativa, negó rotundamente que los alucinógenos enteógenos formasen parte de la cultura de esos pueblos. Para Eliade la lógica nativa, libre de las lógicas tanto alfabéticas como racionales de nuestra sociedad, libraba el inconsciente para producir sueños y visiones de tipo mucho más simbólico y animistas, que lo que podría ser un sencillo estado alucinatorio inducido por una droga. Para dicho autor, lo anterior no corresponde a un ser humano verdadero, sino a un ser humano afectado por un delirio farmacológico. 
Eliade estuvo casi enteramente al mando de la antropología durante décadas. Fue solamente cuando autores como Campbell (1962) y Jung (1969) comenzaron a indagar sobre la mitología y la posible utilización de agentes farmacológicos, tales y como en las naciones antiguas de Asia Menor, el hashish y el opio, entre otras, que se resquebrajó levemente, pero decididamente la tesis fundamentalista y algo severa de Eliade. La llegada de Wasson (1958) produjo en los años cincuenta una profunda perturbación en la tesis de Eliade. Respaldado completamente por antropólogos botánicos como Schultes $(1963,1977)$ y, posteriormente otros autores tales y como Harner (1977) y McKenna (1988), Wasson inició una serie de investigaciones destinadas a comprobar que la base misma de las religiones nativas y chamánicas eran los psicofármacos enteogénicos derivados de las plantas.

Muchos autores siguieron el camino de Wasson para llegar a sugerir que todas las religiones actuales comenzaron en algún punto u otro con una metodología botánica de ingestión enteogénica. Desde luego las exageraciones necesitan darse y proponerse para establecer las polémicas, pues sabemos que en el budismo y el hinduismo de la China y de la India se utilizaban desde la marihuana (para las ceremonias del señor Shiva en los templos) hasta la efedrina (en el té Ma Huan) y el té común negro (teofilina, que es una metilxantina). El primer para entrar en un estado de trance, el segundo y tercero para mantenerse despierto durante las largas horas de oración, contemplación, cánticos y meditación.

La metodología de la conciencia a través de la meditación y el ayuno eran fundamentales a los procesos de alteración de la mente y de su bioquímica cerebral. Wasson -el exagerado, el iconoclasta- fue una bendición para la ciencia de la antropología, y lo es conjuntamente con Schultes (1977), a través de quienes nace la nueva ciencia de la etnopsicofarmacología botánica y antropológica. Poco después, el mundo científico de la botánica y de la antropología comienza a producir innumerables investigadores, que cubren la literatura de estudios muy exactos de los usos tribales de los enteógenos diversos. Wasson (1971) llegó a proponer que el soma, del cual habla el noveno y décimo mándala del Rig Veda, era en realidad el hongo Amanita muscaria. Para un desarrollo reciente de la temática, se recomienda el trabajo de Nichols y Chemel (2006).

El Rig Veda exalta en su noveno y décimo mándala al dios Soma Pavamana, como el que derrota el mal, derrota el sufrimiento y derrota los terribles demonios que traen el dolor. Invoca la presencia del dios Indra que bebe el soma para prepararse para hacer batalla contra lo opaco, lo que carece de valor de iluminación y lo que no contiene salvación. El noveno mándala proclama e invoca al soma para destruir el sufrimiento. Wasson, después de largos estudios sobre el tema, concluyó que el Amanita muscaria era el único elemento y planta enteogénico del soma. También extendió este análisis para incluir el haoma -o sauma- de los Atestas o Avestas, o sea de los textos sagrados de la Persia (equivalentes a los Vedas de la India).

Wasson mantenía que después de la invasión aria de la India, el Amanita muscaria fue introducido en las culturas dravidianas previamente existentes. En ese sentido hubo un cambio en el chamanismo y sacerdocio existente de esa época a nivel profundo. Otros autores como Aldritch (1977), Touw (1981), Sharma (1977) y Quirce, Tyler y Maickel (1988) han mantenido que el soma del Rig Veda era preparado a través del uso del Cannabis sativa.

El Amanita muscaria contiene mucinol y ácido iboténico como ingredientes enteogénicamente activos. Flattery y Schwartz (1989) lograron destruir parcialmente el argumento de Wasson comprobando que el rue sirio o Peganum harmala, un arbusto de las montañas del norte de Asia, era el ingrediente activo del soma. Estos contienen harmalina principalmente como ingredientes 
enteogénicos. Es posible que combinaciones de Amanita muscaria, Cannabis sativa y el Peganum harmala, fuesen todos utilizados para preparar el soma. Incluso existen evidencias muy fuertes que en la India se utilizaba la hierba Argyreia nervosa que produce el ácido lisérgico, un precursor del dietilamida de ácido lisérgico o LSD. Quizás este fuese utilizado para dicho propósito o sea para la preparación del soma. Dichos trabajos sobre la etnopsicofarmacología de la India, muy desafortunadamente, nublaron en parte, la reputación de Wasson y con ello también sus trabajos sobre el hongo teonanacatl (Stropharia cubensis) así como el trabajo extensivo sobre el chamanismo siberiano y el mencionado uso del hongo Amanita muscaria.

\section{Las plantas psicodélicas de las Américas}

Las drogas o plantas enteogénicas alucinatorias del nuevo mundo son muchas. Desde Alaska y el norte del Canadá, hasta la Tierra del Fuego podemos encontrar el uso continuo del tabaco para fines chamánicos de distinto tipos. Ciertamente el tabaco fue utilizado para firmar convenios diversos entre distintas tribus. También para curar en ceremonias y rituales dados. Generalmente, el chamán usaba el tabaco para ayudarse en el transporte a un estado alterado de conciencia enteogénico e invocar los espíritus, dioses y ancestros que servían para efectuar las curaciones diversas. En otros casos, el tabaco era dado a ingerir, mascar o fumar al paciente mismo. La razón de ello está destacada por Dobkin de Ríos (1990), antropóloga norteamericana especializada en la investigación sobre el chamanismo y sus impactos psicoantropológicos en las sociedades antiguas así como las modernas, que mantiene que el tabaco utilizado por los indígenas, contenía altos niveles de harmalina, un alucinógeno enteógeno de actividad moderada y bloqueador de la enzima monoamina oxidasa. La teoría chamánica es que, al transportar el paciente a un estado de felicidad enteogénica por muchas horas, la enfermedad era derrotada por ese estado diferenciado de conciencia, además de ahuyentar a los espíritus de la enfermedad al invocar a los dioses de la salud (la cual es la parte que lleva a cabo el chamán).

Esta diferencia ha sido estudiada en los trabajos de Harner (1977a, 1977b, 1977c), al resaltar el uso diferencial de enteogénicos (hongos y peyote) que se da entre las tribus Mazatecas (Guatemala y México) y los Huichol (norte de México y Estados Unidos). En la tradición Mazateca, el enteógeno (hongos conteniendo psilocibin y psilocibina), es ingerido solamente por el chamán o chamana, tal y como la sacerdotisa/curandera, María Sabina Wasson, Cowan, Cowan y Rhodes (1974). Entonces, bajo el efecto del estado alterado y diferenciado de conciencia -para algunos autores un estado superior de la conciencia- producido por el enteógeno, el médico chamánico hace ceremonias curativas diversas, da discursos de sabiduría y profetiza.

Las ceremonias, como la Velada del Hongo realizado por María Sabina, que fue grabado por (Wasson et al. 1974), duran seis o más horas y son generalmente realizados en los dos idiomas (el indígena y en el castellano). Durante la Velada del Hongo, María Sabina invoca los dioses mazatecas y los santos cristianos similarmente. Los estudios sobre los Huichol han indicado que las ceremonias fueron celebradas ingiriendo el peyote (no los hongos alucinatorios), que contiene la droga alucinatoria y enteogénica mescalina $(3,4,5$ trimetoxi- $\beta$-feniletilamina). La ceremonia tiene un chamán o sacerdote, pero el peyote lo ingieren todos los participantes y todos llegan a tener un estado enteogénico. Autores como Castaneda (1969) mantienen que aparece un guía etéreo que se solía llamar mescalito. Los trabajos de Castaneda han sido muy cuestionados en tiempos recientes por su ausencia de notas de campo antropológicas. Los participantes de la ceremonia del peyote son también llamados la Iglesia Nativa Americana (Native American Church) y tienen autorización en los Estados Unidos para usar el peyote en sus ceremonias religiosas. Es la única iglesia en los 
Estados Unidos autorizada para utilizar un psicofármaco alucinatorio. El vino (alcohol), tabaco, té, café y chocolate, sí están usualmente autorizados.

La diferencia entre las dos organizaciones chamánicas se extiende a través de Centroamérica y partes de Suramérica. Los indios del norte del continente usualmente lograban usar el Sophora secundiflora o frijol del mescal (no contiene mescalina), el Datura stramonium que contiene los alcaloides de la belladona: atropina, hiocinamina y escopolamina, mientras se hizo el extenso uso del Nicotiana tabacum entre las diversas tribus de Norteamérica (también en México, Centroamérica y Suramérica). La posibilidad de entrar en un trance chamánico a través del uso del tabaco -fumado generalmente- es discutido por Dobkin de Ríos (1990), que expone más de cincuenta tribus precolombinas que lo utilizaban para fines que se extienden desde curar el reumatismo, espantar espíritus malignos, desinflamar y curar heridas de insectos y hacer la paz. Hay muchos más fines desde luego, pero el tabaco era considerado una hierba mágica, sagrada y altamente curativa de uso chamánico.

Los indígenas del norte del continente americano no tenían, quizás por el clima, la variedad de opciones enteogénicas de sus hermanos mexicanos o centroamericanos o suramericanos. Aún en los áridos desiertos de Nazca el indígena precolombino disponía de más variedad. Estos incluían el Anandenanthera peregrina, un alucinógeno muy poderoso, el Erythroxylon coca, de donde se deriva la cocaína y el Trichocereus pachanoi, un cactus que contiene mezcalina y es conocido en el Perú como cactus de San Pedro. A estos se le deben añadir el tabaco y muy posiblemente los alcaloides de la belladona. La posibilidad de que en Nazca se utilizara el ayahuasca o yague se debería a la importación y no a su crecimiento botánico, dado el agreste clima desértico. La sobrevivencia para los pueblos de Nazca era muy difícil y su cerámica, iconografía y petroglifos refleja una dependencia muy grande sobre chamanes y dioses. Los cactus alucinatorios y enteogénicos son representados como estrellas. Es posible que las famosas líneas de Nazca sean también un esfuerzo tremendo para atraer lluvia y bendiciones del cielo. Desde luego hay muchas teorías sobre lo anterior.

\section{Precursores del LSD}

Otras drogas enteogénicas que debemos cubrir son las del área mexicana conocida como el Cono Norte, entre otros nombres. La gran diversidad de climas, que van desde lo desértico hasta el bosque lluvioso, nos han dejado bastante número de diversos especímenes botánicos con actividad alucinatoria. Uno de los más interesantes es el llamado ololuiqui o planta serpentina o también comúnmente la campanilla, en inglés morning glory. Dicha planta tiene varias clasificaciones botánicas entre las cuales están las Ipomeas y las Riveas. Una de sus características es que abre sus flores en la madrugada y las cierra a la caída del sol. Por esa razón y dado que son enredaderas, eran asociadas con el dios Quetzalcoatl. Los aspectos de arrastrarse como enredadera por el suelo o los árboles a modo de una serpiente, los elementos enteogénicos como el dios y, el abrir de sus flores, cuando sale el planeta Venus, con la leyenda de la existencia de Quetzalcoatl. En azteca se le llamaba coaxihuitl. Así como las semillas que las plantas de las diversas especies producen, después de muerta la flor, Quetzalcoatl se convierte, con su muerte, en Xochipilli o Señor Dios Príncipe de las Flores. Las flores son los estados enteogénicos, también llamados xochi’s, que contienen la droga amida del ácido lisérgico o LSA -un precursor muy inmediato del LSD, el cual no es producido en la naturaleza- tiene 10 veces menos fuerza alucinatoria que el LSD, lo cual es considerable aún como actividad psicodélica. 
Las semillas de ambas especies -Ipomeas y Riveas- deben de ser molidas cuidadosamente, pues la semilla es dura y no es digerida. Por lo tanto, no suelta su contenido enteogénico a la circulación sanguínea, sin ser triturada. Tanto Ipomeas como Riveas formaban parte de los rituales zapotecas y aztecas y se utilizaban profusamente a través de todo Centroamérica. Como planta enteogénica tanto de utilización mágica como alucinatoria o religiosa, las especies diversas fueron texto y contexto de la civilización precolombina desde México hasta el cono sur. Aún hoy se debate el mecanismo del LSD, pues aunque siempre se ha considerado un agonista del receptor serotoninérgico-2, recientes fuentes de información lo ponen en el dual rol de ser antagonista del receptor 5HT-2 y agonista del receptor 5HT-1 $(5 \mathrm{HT}=$ Serotonina $)$.

El LSA es asombrosamente también producido en el pan de centeno por el hongo infeccioso llamado Claviceps purpurea. La historia del LSA producido a partir del Claviceps purpurea y el pan de centeno debe merecer de nuestra parte una mención, pues constituye parte del chamanismo europeo y de su socioeconomía. Las brujas de Europa eran originalmente parteras, enfermeras y chamanes y fueron perseguidas porque estaban compitiendo la clientela de los médicos del medioevo a través de su herbología y de sus obras con enfermos y mujeres embarazadas. Aún muchas aristócratas acudían a ellas en busca de medicamentos herbales. Entre sus drogas estaba la ergotamina, que es un alucinógeno también producido por el hongo Claviceps purpurea. Se usa además para bien parir. Entre otros enteógenos conocían el Amanita muscaria, la escopolamina, la atropina y la hiocinamina -es posible que utilizasen aún otros que con las persecuciones se llegaron a perder.

Cuando la humedad era alta en Europa, y entre las clases más pobres, que usaban pan de centeno y no de trigo, el Claviceps purpurea era más activo. Aldeas enteras bailaban desenfrenadamente a veces el baile de San Vito. Matossian (1989), mantiene que muchos de los estados místicos reportados en el medioevo en los monasterios y conventos, también reflejan el grado en que el voto de pobreza obligaba a los monjes y monjas (frailes, etc.) a comer el pan de centeno. Cuando el clima era propicio (alta humedad), el Claviceps purpurea invadía el pan y producía ergotaminas y LSA. Las brujas que no pertenecían al establecimiento eran quemadas, más la burguesía cuyos miembros habitaban en los monasterios, eran alabados. Para Matossian el principio activo era el mismo en uno u otro caso. Incluso muchos de los casos reportados de licantropía y vampirismo durante el siglo XIX, puede que reflejen cambios drásticos en la economía del campesinado, que pasaba del pan de trigo al de centeno. Quizá a la base misma de la novela de Bram Stocker "El Conde Drácula" esté un sencillo hongo del centeno.

\section{Plantas chamánicas de los aztecas}

Otros elementos importantes de la vida enteogénica de las Américas precolombinas son los hongos que contenían psilocibin y psilocibina. Fundamentales a los rituales religiosos y mágicos de casi todas las naciones mexicanas precolombinas, y en particular los aztecas, provenían de los géneros de Psylocibe y Stropharia. De todas las 15 o más especies de hongos alucinatorios que se han identificado solamente en el área mexicana, el Stropharia cubensis es el más poderoso en actividad y efecto alucinatorio. Generalmente, el efecto del psilocibin y de la psilocibina está basado en un agonismo del receptor serotoninérgico 5HT-2.

Muchos de los teoremas del llamado déficit en gating desarrollado por Geyer (1998) y Geyer y Vollenweider (2008) entre otros, se han desarrollado alrededor de dicho agonismo serotoninérgico. Los aztecas solían recoger los hongos amarillos en la noche y comerlos crudos. No los cocinaban, 
ni tampoco los solían secar. El principal hongo de los aztecas era llamado por ellos teonanacatl, que se traduce como la carne o piel de dios. Los españoles consideraban que tal nombre indicaba claramente que era una parodia blasfema de la santa misa y del sacramento de la eucaristía. Hubo persecuciones poderosas y los aztecas se les prohibieron usarlo. Los templos sagrados fueron destruidos como recintos del diablo. Todo esto estuvo basado en una mala traducción del azteca al castellano. El hongo produce un sentimiento físico de energía que corre por la piel. La carne de dios era en realidad, en azteca, el efecto que tiene el hongo (enteógeno) sobre la piel. Una mala traducción convenció a los frailes de la existencia de un pacto demoníaco y una civilización fue destruida. No sabemos si Cortés se enteró de dicha traducción, pero ciertamente fue muy conveniente para la militar española el que fuese traducido en forma errada a los frailes.

Algunos de los hongos de México enteogénicos/alucinatorios son el Conocybe siligineoides, el Panaeolus sphintrinis, el Psylocibe cautísima, el Psylicibe aztectorum, etc. Como habíamos ya mencionado el más poderoso es el Stropharia cubensis. Los aztecas también utilizaban además del peyote y las Ipomeas o Riveas, una serie de Daturas llamados en Azteca toloache, generalmente derivados de la Datura stramonium y también conocido como el toloatzin. Hay muchos distintos tipos de Brugmansia, generalmente en Costa Rica se conocen como las distintas variedades de la reina de la noche. El principal alcaloide de la Brugmansia es la escopolamina. En Europa se utilizó en vez de las Brugmansias, el Atropa belladona, la Mandragora officinarum y el Hyocyamus nigez, particularmente en los aquelarres de las brujas. Así pues, las cuatro drogas de los aztecas eran el olioluqui, el peyote, el teonanacatl y toloache. También puede haber existido el Salvia divinorum como una de ellas. Generalmente existía una cultura popular del uso de los enteógenos, más otra cultura mágico religiosa, preparada con fórmulas secretas por los sacerdotes representantes de la teocracia azteca.

\section{Plantas alucinatorias de las Amazonas}

Las plantas alucinatorias principales de las Amazonas son las que contienen triptaminas, alcaloides de la Datura, beta carboxilasas y nicotina. Existen muchas más de acuerdo con las tradiciones chamánicas, pero no son aun conocidas ni botánicamente ni farmacológicamente. Algunas de dichas plantas se centran alrededor de derivados de la dimetiltriptamina, son tomados o utilizados en polvo que se absorbe por la nariz como un rapé, en algunos casos son fumados. De los principales utilizados están los Virola, entre los cuales destaca la Virola theiodora (existen más de cincuenta especies del género Virola en las Amazonas).

Los indígenas de las Amazonas del noroeste, particularmente en el Brasil y Colombia, además de la región del Orinoco en Venezuela, utilizan la resina de diversos árboles de la especie Virola a modo hedónico y ceremonioso. La resina es calentada en fuegos lentos hasta cobrar un color rojo oscuro y éste es luego convertido en un polvo utilizado en forma de rapé (Schultes \& Hofman, 1980). Dicho rapé se conoce como yakee y yato en Colombia y paricá, epená y nyakwana en Brasil. Muchos indígenas residentes de los Vaupés colombianos (Barasana, Makuna, Puinave, Kabuyarí, Kuripako) le quitan la corteza al árbol de la Virola antes de que el sol caliente el tronco del árbol y le quitan la corteza con su exudación resinosa roja. Los pedazos de corteza son luego cortados en pedacitos y hervidos hasta obtener un sirope espeso. Cuando el sirope ha sido secado al sol, es pulverizado y mezclado con las cenizas de la corteza de otra especie salvaje de Theobroma subinucanum. El rapé resultante es tan poderoso que Schultes y Hofman (1980), reportan que ha inducido la muerte de chamanes brujos ya viejos. 
Para Biocca (1966), las otras especies utilizadas en la manufactura de rapés, además de Virola theiodora, son la $V$. calophylloidea, $V$. cuspidata y $V$. rufula. Los ingredientes activos de índole alucinatorio de los Virola son la dimetiltriptamina (DMT), la N-metiltriptamina (MMT), la 5-metoxi- $N, N^{\prime}$-dimetiltriptamina (5-MeODMT), la 5-metoxi- $N$-metil triptamina (5-MEO-MMT), la 2-metil-6-metoxi-1,2,3,4-tetrahidro-beta-carbolina (6-MeO-THC), y la 1,2 Dimetil1-6-metil1,2,3,4-tetrahidro-beta-carbolina (6-MeO-DMTTHC). Todos, de acuerdo con Schultes y Hofman (1980), son alcaloides y dos de ellos, que contienen beta-carbolinas, actúan como inhibidores de la monoamina oxidasa (MAO), enzima que degrada las aminas biogénicas. Por lo tanto, el efecto de dichos rapés, puede durar por horas, sin que las triptaminas sean degradadas por la enzima monoamina oxidasa.

Otras plantas utilizadas en polvos alucinatorios de tipo rapé en las Amazonas son las Anandenantheras tanto la $A$. columbrina como la $A$. peregrina. La $A$. peregrina es también conocida con el nombre botánico de Piptadenia peregrina. El polvo/rapé se prepara a partir de los frijoles de la planta en el Valle del Orinoco. Schultes (1998) refiere que en tiempos de la preconquista era utilizado en las Indias Occidentales. El autor indica, que los reportes europeos más tempranos sobre el uso de la droga, llamada por los indios cahoba, datan de 1496. Los indios Taino del Caribe lo solían inhalar para comunicarse con el mundo de los espíritus (Schultes \& Hofman, 1980). E1 mapeo del uso de la $A$. peregrina en la forma de rapés incluye todo el Valle del Orinoco y partes del sur de Venezuela, el oeste de los Andes colombianos norteños y mucho del Valle de la Magdalena, e incluye además Colombia, Ecuador, Perú y Bolivia, bajando de los Andes y la costa de Perú. Se han notado algunas regiones del norte de Argentina en que se utilizan también.

Los polvos rapé se conocen con los nombres vilca o huilca, cébil y cahoba. También en Brasil se utiliza el nombre paricá. Aun otro nombre es yopo. Se debe tomar en cuenta que los indígenas a menudo mezclan otras hierbas o frijoles o raíces en sus formularios para obtener efectos más poderosos. Al respecto de los compuestos químicos de los Anandenanthera son los mismos que los de los Virola, con la excepción de la bufotenina que está presente en ellos (Schultes \& Hofman, 1980). La descripción química de la bufotenina es el 5-hidroxi- $N$, $N^{\prime}$ - metiltriptamina (5-OHDMT). Las bufotenina siempre ha sido controversial al respecto de sus efectos alucinatorios.

Ayahuasca o yagué o hoasca es una mezcla de bejucos que crecen en la selva lluviosa amazónica. El más simple es en parte Banesteriopsis caapi mezclado y cocido con la planta conocida con el nombre botánico de Psychotria viridis. La base de su efecto enteogénico es la dimetiltriptamina o DMT y la harmalina. La harmalina procede del Banesteriopsis caapi y el DMT viene de la Psychotria. El Banesteriopsis tiene como 100 distintos géneros de bejucos. Se reconoce en el Amazonas con el nombre de caapi, pinde, natéma y yajé, además de ayahuasca o hoasca. Se utiliza la corteza de los tallos del bejuco en tanto el Brasil, Bolivia, Colombia, Ecuador, Perú, el Orinoco Venezolano y la costa pacífica de Colombia.

Existen dos especies de Banisteriopsis utilizados con mayor frecuencia, el ya mencionado Banesteripsis caapi y además el Banisteriopsis inebrians. Sus ingredientes activos son la harmina, el harmol, la harmina-N-oxido, la harmalina, el ácido harmalínico, el tetrahydroharmian y la kertotetrahidro-norharmina. El Banisteriopsis rusbyana contiene también dimetiltriptamina (DMT), al igual que el Psychotria viridis. El Psychotria viridis y el P. carthaginensis se utilizan ambos en la forma de hojas con la corteza del bejuco del Banisteriopsis caapi. Son ambos utilizados por los indígenas Sharanahua y Culima en el Amazonas del suroeste. Sus ingredientes activos son la N-metiltriptamina y la N-metil-tetrahidro-beta-carbolina, además de la $N, N^{\prime}$-dimetiltriptamina. 
De los hechos etnopsicofarmacológicos más interesantes del chamanismo selvático resalta esta combinación. Lo anterior es debido al hecho que el DMT es altamente sensible a ser metabolizado por la enzima monoamina oxidasa (MAO), principalmente. Por lo tanto no dura mucho tiempo en el cerebro y la intensidad del efecto enteogénico/alucinatorio es muy pasajero. Más, si se combina el Psychotria viridis con el Banisteriopsis caapi, esto cambiará. Esto es debido al sencillo hecho de que el Banisteriopsis contiene harmalina, que además de ser un alucinógeno débil, logra impedir el metabolismo del alucinógeno más poderoso, que es el DMT, pues bloquea la enzima MAO. Ello da lugar a un largo periodo de un estado enteogénico a nivel experiencial (ya que no es degradado enzimáticamente la dimetiltriptmina).

¿Cómo pudieron los chamanes selváticos del Amazonas conocer esto? ¿Fue por tanteo y error o por otro medio del conocimiento? Por tanteo y error hubiese sido similar a buscar una aguja en un pajar. Muchos de estos aciertos del chamanismo son asombrosos. Al menos, siguiendo las leyes de la probabilidad estadística, pareciera ser que el chamanismo usó algo más que los sistemas de tanteo y error. Uno de las características de la ayahuasca es la intensidad y duración de la experiencia alucinatoria. Otro tipo de bejuco que parece jugar un papel importante en la preparación de la ayahuasca es el Tetrapteris methystica (Schultes \& Hofman, 1980).

\section{Rituales y ceremonias}

Para Miller (1983) y Quirce y Maickel (2009), el ritual genera cambios neurofisiológicos que interactúan con la droga ingerida. Las horas de preparación a través de la danza, los cánticos, las invocaciones, etc., constituyen también la base de los cambios observados con el enteógeno mismo. El trabajo de Winkelman y Balser (2008) hace mención de la relación en lo ritual y lo neurofisiológico. No es de extrañar que parezca sobrenatural, el que hongos, semillas alucinatorias y bejucos cambien el curso de una enfermedad, si postulamos que exista en el cerebro y en la mente una neurofisiología de transformación, que puede ser afectada a través de dichos rituales y dichas drogas. Desde luego, nos encontramos aún en el umbral experimental de lograr este tipo de investigación. Más, el chamanismo lo ha estado practicando por milenios. Aun buscamos como científicos, solamente la droga milagrosa, sin aún darle importancia suficiente a la estimulación neuromental, que los rituales producen e inducen en las personas.

El reduccionismo científico a veces peca de ausencia de análisis holístico en el mismo diseño experimental que utiliza. La mayor parte de la experimentación ha estado orientada a encontrar el ingrediente activo en el formulario herbal -y no en el hombre completo- que estimula su cerebro y su mente por diversos medios. Ambos son necesarios para lograr una mayor comprensión de la curación chamánica. De 17 tribus examinadas en su uso de enteogénicos a través de las Américas, todos utilizaban la danza, el canto y la música con ritmos alternantes y continuos, que duraban horas y que inducían trances hipnóticos en los participantes. Este estudio citado en Dobkin de Ríos (1990), incluyó los Tukamo de Colombia, los Yanomano de Venezuela y Brasil, los Aztecas de México, los Luiseños de California, los Teneteharas de Brasil, los Mestizos de Perú, los Navajo de los Estados Unidos, los Chama de las Amazonas, los Cashinahua del Amazonas peruano, los Huichol de México, los Mazatecas de México, los Jívaro del este del Ecuador, los Sharanahua y los Culima de las Amazonas peruanas, los Amahuaca de las altas Amazonas y los Kiowa y Comanches de los Estados Unidos. El estudio diferencia entre distintos enteógenos utilizados en largas ceremonias. 


\section{Teorías antropológicas sobre el chamanismo}

Quizás unas de las propuestas más interesantes que se han realizado en los últimos 30 años son las que corresponden a la obra de Jaynes (1976). Dicho trabajo intenta, en forma muy ambiciosa, llegar a establecer una verdadera antropología neurofisiológica a nivel histórico y cultural. El argumento fundamental de Jaynes es que, hace 3500 años, se dio un quiebre en las estructuras culturales e históricas del mundo antiguo, lo que hoy conocemos como el mundo moderno. Dicho quiebre responde a un giro de la dominación del hemisferio cerebral derecho sobre el izquierdo a una predominancia del izquierdo sobre el derecho. Para Jaynes, el hombre era aquel que tenía una mayor dominación del hemisferio derecho sobre el izquierdo. Jaynes describe las características de la dominación del hemisferio derecho sobre el izquierdo en términos de cinco puntos básicos: (a) Espacialización, adonde las dimensiones del espacio y del tiempo se estiran, (b) indicación selectiva, adonde se escogen solamente ciertos elementos del territorio del mapa de la mente, (c) la creación de un yo y un $m i$ de tipo analógico, (d) narratización, adonde los eventos son seleccionados por su congruencia y desarrollo secuencial y (e) conciliación, adonde las experiencias son concientemente asimiladas el uno al otro.

Hubo un cambio que produjo el quiebre mencionado y el principio de la dominación del hemisferio izquierdo sobre el derecho. La razón de este cambio, reside para Jaynes en una catástrofe que sufrió el mundo antiguo debido a la erupción del volcán Thera en 1500 a.C. Dicha erupción destruyó casi toda la cultura del mundo antiguo y hubo que reestructurarlo de nuevo. La reestructuración necesitó del invento de los alfabetos modernos y esto forzó el aprendizaje verbal del hemisferio izquierdo como hecho predominante de la cultura. Antes de ese evento, si bien había escritura, la cultura era trasmitida por el oír y no el leer. Después comienza a establecerse la dominación del hemisferio izquierdo sobre el derecho, hasta llegar a nuestra presente civilización.

Siguiendo dicha lógica, bien puede ser que nuestros antepasados precolombinos fuesen utilizadores preferenciales del hemisferio derecho y los europeos que llegaron lo eran del izquierdo. Para la presente tesis basta decir que Jaynes mantiene que, anteriormente a esos 3500 años, el hemisferio derecho era ejecutivo y que pasaba órdenes al hemisferio izquierdo, desde los centros alucinatorios del cerebro hacia el área de Wernicke y estas órdenes eran luego desglosadas por el área de Broca que alucinaba las órdenes del hemisferio derecho para construirlas en voces celestiales. Por ende, se lograba construir las civilizaciones de los reyes dioses, adonde el hemisferio izquierdo le daba un rol de profeta o rey o mensajero elegido por dios a la persona que en base a eso se creía que recibía órdenes celestiales.

El hemisferio derecho respondía a una lógica cultural mucho más mitológica que la del izquierdo. Ya en el tiempo de la Iliada y de la Odisea, se encuentran, para Jaynes, un Homero que presenta las dos opciones: hombres dominados por dioses y hombres más libres en proceso de elaborar sus propias mentes. Más, el paraíso de hombres y dioses perdido por la catástrofe universal del volcán Thera no había de volver y aquellos adanes tendrían que labrar sus mentes con sus esfuerzos para alcanzar un nuevo cielo. Una tesis que adelanto en este artículo es que los sacerdotes de la vieja religión pre-catástrofe posiblemente se convirtiesen en los chamanes de la nueva.

Aun otra tesis que adelanto es que el uso de alucinógenos y enteógenos que no menciona Jaynes como factor, existía en ese tiempo profusamente (en eso Jaynes se parece también a Eliade, 1966). Es posible que el uso de alucinógenos y enteógenos sobreestimulara el hemisferio derecho y llegara a alucinar el izquierdo para crear, así las grandes religiones mágico alucinatorias de los 
dioses reyes que menciona Jaynes en su obra. También, adelanto un corolario obvio y lógico de dicha tesis, por cuanto que el mundo antiguo posiblemente perdiese con la catástrofe y durante siglos, las conexiones básicas con la India y con la China de adonde provenían, el opio (Papaver somniferum), la marihuana (Cannabis sativa) y el rue sirio (Peganun harmala), etc.

Es posible que también y al entrar en catástrofe, el conocedor y sabio sacerdocio de las sociedades bicamerales, rico en artes avanzadas de una farmacopea enteogénica, perdiesen las fórmulas más eficientes en el uso de enteógenos y otros psicoherbarios. Por ende, se perdería en gran parte los sistemas elaborados por aquellas culturas para sobreestimular el hemisferio derecho y alucinar el izquierdo. Muchos sistemas de meditación hoy predican el retorno a estados paradisíacos de paz y tranquilidad. Deben de haber habido muchos de estos, que se perdieron al morir las civilizaciones debido a la gran catástrofe volcánica. Quizás solamente sobrevivieron los conocimientos de aquellos sacerdotes entonces convertidos en chamanes o brujos de la nueva era.

El chamanismo era mucho más antiguo, como dijimos originalmente. Data desde los periodos neolíticos y paleolíticos. Ciertamente como apunta Pokorny (1970), las plantas alucinatorias que el chamanismo diferencia y utiliza, se encuentran dibujadas en las cuevas del paleolítico y neolítico. Más, la catástrofe posiblemente devolvió al chamanismo muchos elementos de una clase que otrora tiempo fuese sacerdotal. Volviendo a nuestras Américas precolombinas, se repite la catástrofe en otra forma, pues de nuevo civilizaciones y culturas enteras basadas en el uso de enteógenos para sus fines religiosos y de curación, y por ende, que posiblemente fuesen orientadas hacia una predominancia del hemisferio derecho sobre el izquierdo, cayeron ante los proponentes de una lógica de pensamiento y aprendizaje, ideología y religión, basada en la predominancia del hemisferio izquierdo, el fin, pues, de las grandes religiones mágicas/alucinatorias y enteogénicas de las Américas. El hemisferio de la escritura y el hemisferio de la fantasía aun no se han logrado de nuevo unir en la civilización y en la cultura planetaria. En la India, muchos de estos problemas se llegaron a llamar maya o estados ilusivos.

Como nuevas teorías antropológicas del chamanismo son fundamentales mencionar a tanto McClenon (1997) y a Winkelman (2000). El primero ha mantenido el que los rasgos de curación y de facilidad de entrar en estados hipnóticos o de trance, así como de lograr tener estados alterados y superiores de la conciencia, es parte de una heredad genética a partir del principio de la existencia de Homo sapiens sapiens en la forma de Cro-Magnon. McClenon no niega la existencia de otros factores tales como lo son el entrenamiento de las personas en estrategias y técnicas de la conciencia, más, considera que el rasgo genético es el predominante y que este separó desde el tiempo de las cavernas al chamán del resto de la humanidad.

Además, Winkelman (2000) ha indicado que dichos rasgos de curación, facilidad de tener experiencias hipnóticas y alteradas de tipo superior y diferenciado, corresponden al grado de entrenamiento de la persona en su vida con técnicas de mejoría espiritual y físico/mental. Así pues mantiene que las personas que desarrollan rasgos chamánicos, lo hacen debido al uso durante largos periodos de una tecnología de la conciencia de tipo meditativo, contemplativo, de oración, ayunos y dietas inteligentemente diseñadas, repetición de mantras y uso de mándalas, lecturas de tipo enaltecedor, reflexiones filosóficas y teológicas, uso inteligente de la herbología y uso igualmente inteligente de sustancias alucinatorias, etc. Dichas personas son más sensibles, más, el uso de dichas metodologías aumentan la sincronización entre el hemisferio izquierdo y el derecho del cerebro. Para Winkelman, dicha sincronización eventualmente llega a establecer nuevos rasgos entre los cuales está la capacidad de curar a otras personas. 
Algunos de estos estudios de sincronización ya se habían realizado en los años 70 con la meditación trascendental y se utilizaron mediciones electroencefalográficas. Más recientemente, Orme-Johnson, Schneider, Son, Niddich y Cho (2006), han realizado estudios de tomografía al respecto de los efectos de la meditación trascendental, en lo que respecta a la reactividad del cerebro ante el dolor.

Mohandas (2008) ha realizado un excelente resumen tomográfico de las áreas cerebrales activadas por diversos métodos de meditación y oración, y debería leerse. De dicho trabajo y otros similares, se desprende que, en realidad, el chamanismo también difiere entre sí, dependiendo del uso de la metodología de inducción de alteración, diferenciación y obtenimiento de una conciencia superior. Ello a su vez indica que, aunque McClenon tenga razón, existe una variedad neurocerebral de inducción de estados de conciencia alterna, alterada y superior, que es muy grande.

La existencia de un ser supremo en la historia y la antropología humana está muy ligada a la existencia de estados superiores de la conciencia. Desde las tradiciones monásticas del Asia y del Occidente hasta las tradiciones chamánicas de los pueblos aborígenes, la experiencia de dios parece ser una experiencia transpersonal de la máxima importancia en la comprensión de lo que es un estado alterado y superior de la conciencia.

Winkelman y Balser (2008) enfatizan que la religión y el culto/veneración del maestro es un derivado de los rituales animales de sumisión/dominación. Los autores, sí, evitan caer en un reduccionismo sobre el modelo animal e indican que el hombre difiere a su vez del animal en su experiencia y su antropología religiosa. Ernandes y Giammanca (1998) han propuesto el teorema de que el culto de dios nace en tiempos antiguos como consecuencia de un desafuero en la relación en el cerebro trino. Este consiste en las tres partes conocidas como la corteza cerebral, adonde ocurre gran parte del conocimiento y la cognición, el cerebro paliomamífero o el sistema límbico, adonde ocurren y son mediatizadas gran parte de las emociones y el sistema reptiliano que tiene numerosas funciones autonómicas y que ante una amenaza concluye que se trata de seres de la misma especie.

El sistema límbico tiende a interpretar las amenazas, como problemas derivados y asociados a la dominación (de otras personas de la misma especie). Ernandes y Giammanca mantienen que en tiempos primitivos, la neocorteza sufrió un trauma poderoso al llegar al conocimiento de que existía la mortalidad. Dicho "choque existencial" afectó el sistema límbico, y tanto la neocorteza como la corteza paliomamífera disminuyeron su usual inhibición del sistema reptiliana. Por ende, este último envió el mensaje a la corteza frontal de que se trataba de un individuo dominante y la corteza tuvo que crear la existencia de un ser de enorme poder que regulaba la muerte del sistema. Consecuentemente, se llegó a crear un ser de la misma especie que era omnipotente. De ahí que el concepto de dios se propagase en los tiempos antiguos. Dicho ser se convirtió en merecedor de ceremonias, rituales, oraciones y toda otra conducta humana de sumisión.

Desde luego, dicho teorema ha de investigarse con mayor cuidado a nivel antropológico. Sí, encabe en el pensamiento de algunos antropólogos y pensadores modernos, como Hancock (2007), el mantener que hace 50.000 años hubo una explosión de creatividad artística en las pinturas de las cuevas y de creación de estatuas tanto religiosas como de otro tipos. Se mejoró el armamento y se encuentran pulseras y otros adornos en los yacimientos de dichos hombres. Esto ha sido rebatido muy recientemente por otros antropólogos (Diller \& Cannon, 2009; Henshilwood, d'Errico, Marean, Milo, \& Yates, 2001; McBreaty \& Brooks, 2000; Mellars, Boyle, Bar-Yosef, \& Stringer, 2007) que mantienen que hace 80.000 años se encontraban en el África también dichos adornos. 
Naturalmente la hipótesis de Ernandes y Giammanca (1998) encabe en cualquier momento en que existan vacíos en la creatividad religiosa del hombre antiguo y, de un momento a otro, estalle la pintura religiosa y el arte orientado a dios en sus muchas formas antiguas. Por otra parte, Winkelman y Balser (2008) indican que la especie Neandertal (Homo neanderthalensis) era también religioso dado el culto de difuntos que se ha encontrado en sus cuevas.

Entre los trabajos más completos que recientemente han sido publicados está el muy reciente trabajo de Fingelkurts y Fingelkurts (2009), que busca diferenciar a través de la literatura científica la relación entre dios como lo que el cerebro crea y dios como lo que la mente/cerebro llegan a percibir. No derivan dichos autores conclusiones finales al respecto, pero exponen una variedad de literatura muy vasta y de excelente calidad. Sí, indican la necesidad de llevar a cabo mayor experimentación investigativa en dicha área y señalan los aspectos transpersonales de la experiencia religiosa que siempre es reportada como algo fuera de sí mismo que, a su vez, otorga significado a la persona. Fingelkurts y Fingelkurts (2009), sí, indican que la experiencia religiosa coexiste tanto con la esquizofrenia como sin ella. Nichols y Chemel (2006) exponen en su reciente trabajo la relación entre chamanismo y el uso de diversos agentes psicofarmacológicos.

\section{Conclusiones}

El mapeo psicofarmacológico, enteogénico y antropológico de Costa Rica yace aún por llevar a cabo. Si bien sabemos que nuestros antepasados precolombinos utilizaban también agentes alucinatorios, no se ha hecho aun nada por identificar la botánica enteogénica del país. Esto es algo que debe llevarse a cabo en el futuro y en el presente que elabora ese futuro. Eso es también lo que pretendemos llevar a cabo en nuestro programa de Psicobiología y Psicofarmacología de la Escuela de Psicología en colaboración con la Facultad de Farmacia en la Universidad de Costa Rica.

\section{Referencias}

Aldritch, M. R. (1977). Tantric cannabis use in India. Journal of Psychedelic Drugs, 9, 227-233.

Biocca, E. (1966). Viaggi tra gli indi alto Rio Negro alto Orinoco. Consiglio Nazionale delle Recherche, 2, 235-252.

Campbell, J. (1959). Primitive Mythology. New York: Viking Press.

Campbell, J. (1962). Oriental Mythology. New York: Viking Press.

Castaneda, C. (1969). The Teachings of Don Juan: A Yaqui Way of Knowledge. Los Angeles: University of California Press.

Diller, K. C., \& Canon, R. L. (2009). Evidence against a genetic based revolution in language 50.000 years ago. En R. Botha, \& C. Knight (Ed.), The cradle of language studies in the evolution of language. New York: Oxford University Press.

Dobkin de Ríos, M. (1990). Hallucinogens: cross-cultural studies perspectives. Prospect Height, Illinois: Waveleand Press.

Eliade, M. (1966). Shamanism: archaic techniques of ecstasy. New York: Pantheon Books.

Ernandes, M., \& Giammanca, S. (1998). Maclean's triune brain and the origin of "immense power being" idea. Mankind Quaterly, 39, 173-199.

Fingelkurts, A. A., \& Fingelkurts, A. A. (2009). Is our brain hardwired to produce Gods or is our brain hardwired to perceive God? A systematic review of the role of the brain in mediating religious experience. Cognitive Process, 10, 293-326. 
Flattery, D. S., \& Schwartz, M. (1989). Haoma and harmaline: the botanical identity of the IndoIranian sacred hallucinogen "soma" and its legacy in religion, language and middle eastern folklore. Berkeley, California: University of California Press.

Geyer, M. A. (1998). Why study hallucinogenic drugs in animals? The Heffter Review of Psychedelic Research, 1, 333-339.

Geyer, M. A., \& Vollenweider, F. X. (2008). Serotonin contributions to understanding psychosis. Trends in Pharmacological Sciences, 29, 445-453.

Harner, M. J. (1977a). Introduction. En M. J. Harner (Ed.), Hallucinogens and shamanism. London: Oxford University Press.

Harner, M. J. (1977b). The sound of rushing water. En M. J. Harner (Ed.), Hallucinogens and shamanism. London: Oxford University Press.

Harner, M. J. (1977c). Common themes in South American Yagé experiences. En M. J. Harner (Ed.), Hallucinogens and shamanism. London: Oxford University Press.

Henshilwood, C. S., d'Errico, F., Marean, C. W., Milo, R. G., \& Yates, R. (2001). An early bone tool industry from the middle stone age at Blompos Cave, South Africa: implications for the origin of modern behaviour, symbolism and language. Journal of Human Evolution 41, 631-678.

Jaynes, J. (1976). The origin of consciousness and the breakdown of the bicameral mind. Boston: Hougton Mifflin Co.

Jung, C. (1969). Psychology and religion. New Jersey: Bollingen Series XX, Princeton University Press.

Matossian, M. K. (1989). Poisons of the past: molds, epidemics and history. New Haven, CT: Yale University Press.

McBreaty, S., \& Brooks, A. S. (2000). The language that wasn't: a new interpretation of the origin of modern human behaviour. Journal of Human Evolution, 39, 543-563.

McClenon, J. (1997). Shamanic healing, human evolution, and the origin of religion. Journal for the Scientific Study of Religion, 36, 345-354.

McKenna, T. (1988). Hallucinogenic mushrooms and evolution: revision. The Journal of Consciousness and Change, 10, 4-11.

Mellars, P., Boyle, K., Bar-Yosef, O., \& Stringer, C. (2007). Rethinking the human revolution. New behavioural and biological perspectives on the origin and dispersal of modern humans. Great Britain: McDonald Institute for Archeological Research.

Miller, R. A. (1983). The magical and ritual use of herbs. California: Inner Traditions.

Mohandas, E. (2008). Neurobiology of spirituality. En A. R. Singh, \& S. A. Singh (Eds.), Medicine, mental health, science, religion and well-being 6, (1), 63-80. California: Men Sana Monographs.

Nichols, D.E., \& Chemel, B.R. (2006). The neurophar-macology of religious experience. En: P. McNama-ra (Eds.) Where God and Science Meet. How Brainand Evolutionary Studies Alter our Understanding of Religion (pp. 1-34) Chap. I, Vol. 3.

Orme-Johnson, D. W., Schneider, R. H., Son, Y. D., Niddich, S., \& Cho, A. H. (2006). Neuroimaging of meditation effect of brain reacivity to pain. Neuroreport, 17, 1359-1363.

Ott, J. (1993). Pharmacoteon: Entheogenic drugs, their plant sources and history. Kenniwick, WA: Natural Products Co. 
Pokorny, A. (1970). The hallucinogens in anthropology, prehistory and the history of the plastic arts. Prague: $7^{\text {th }}$ Congress of the Collegium Internationale Neuro-Psychoparma-Cologium, 7, 16-18.

Quirce, C. M., \& Maickel, R. P. (2009). Los alucinógenos y los teoremas del “gating” en la acción psicofarmacológica alucinatoria y en la esquizofrenia. Revista Costarricense de Psicología, 28, 41-42, 85-99.

Quirce, C. M., Tyler, V., \& Maickel, R. P. (1988). Concepciones culturales de los estados alterados de la conciencia. Neuroeje, 6, (1), 15-30.

Schultes, R. E. (1963). Hallucinogenic plants of the New World. The Harvard Review, 1, 18-32.

Schultes, R. E. (1977). Mexico and Columbia: two maya centers of aboriginal use of hallucinogens. Journal of Psychedelic Drugs, 9, 173-176.

Schultes, R. E. (1998). Antiquity in the use of New World hallucinogens. The Heffter Review of Psychedelic Research, 1, 1-7.

Schultes, R. E., \& Hofmann, A. (1979). Plants of the gods: origins of hallucinogenic use. Rochester: Healing Arts Press.

Schultes, R. E., \& Hofmann, A. (1980). The botany and chemistry of hallucinogens. Illinois: Charles C. Thomas.

Sharma, G. K. (1977). Ethnobotany and its significance for Cannabis studies in the Himalayas. Journal of Psychedelic Drugs, 9, 937-939.

Touw, M. (1981). The religious, medicinal and uses of Cannabis in China, India and Tibet. Journal of Psychoactive Drugs, 13, 23-34.

Wasson, R. G. (1958). The divine mushroom: primitive religion and hallucinotory agents. Proceedings of the American Philosophical Society, 102, 221-223.

Wasson, R. G. (1971). Soma of the Rig Veda: what was it? Journal of the American Oriental Society, 91, (2), 169-187.

Wasson, R. G., Cowan, G., Cowan, F., \& Rhodes, W. (1974). María Sabina and her Mazatec mushroom velada. New York: Harcourt Brace Jovanovich.

Winkelman, M. J. (2000). Shamanism: the neural ecology of consciousness and healing. Westport, CT: Bergin, \& Garvey.

Winkelman, M. J., \& Balser, J. (2008). The supernatural as natural: biocultural approach to religion. New Jersey: Pearson Prentice Hall.

\section{Reseña del autor}

\section{Carlos M. Quirce Balma}

Bachiller en Química, Master en Psicología y Doctorado en Farmacología, Posdoctorado Universidad de Purdue. Es catedrático e investigador en la Escuela de Psicología y en la Facultad de Farmacia de la Universidad de Costa Rica. Obtuvo el Premio Internacional de Radiofarmacología en 1981, Premio Fulbright en 1982, posee diversos doctorados honoris causa; miembro distinguido del Colegio Profesional de Psicólogos de Costa Rica, mención honorífica Revista Científica del Colegio Profesional de Psicólogos de Costa Rica, miembro emérito de la Academia de Ciencias de Nueva York, mención de trayectoria de vida del Colegio Profesional de Psicólogos de Costa Rica. Profesor homenajeado en el año 2011 por la Universidad de Costa Rica. 